\title{
Combining immunotherapy and radiation therapy for small cell lung cancer and thymic tumors
}

\author{
Suchit H. Patel ${ }^{1}$, Andreas Rimner ${ }^{1}$, Roger B. Cohen ${ }^{2}$ \\ ${ }^{1}$ Department of Radiation Oncology, Memorial Sloan Kettering Cancer Center, New York, USA; ${ }^{2}$ Division of Hematology-Oncology, Perelman \\ Center for Advanced Medicine, Philadelphia, USA \\ Contributions: (I) Conception and design: SH Patel, A Rimner, RB Cohen; (II) Administrative support: A Rimner, RB Cohen; (III) Provision of study \\ materials or patients: None; (IV) Collection and assembly of data: None; (V) Data analysis and interpretation: None; (VI) Manuscript writing: All \\ authors; (VII) Final approval of manuscript: All authors. \\ Correspondence to: Andreas Rimner, MD. Department of Radiation Oncology, Memorial Sloan Kettering Cancer Center, 1275 York Avenue, New \\ York, NY 10065, USA. Email: rimnera@mskcc.org.
}

\begin{abstract}
Recent work with immunotherapy has shown promising results with treatment of several solid malignancies, and there are several reports of good systemic responses with the combination of immunotherapy and radiation therapy (RT), most notably in advanced melanoma. Given the rapid increase in the use of checkpoint blockade as well as anti-tumor vaccines, we review here the preclinical rationale and ongoing clinical work in combining immunotherapy with RT for small cell lung cancer (SCLC) and thymic tumors. While there are several reports of promising results with the combination of immunotherapy and conventional systemic treatment, we focus here on the ongoing clinical studies that combine immunotherapy with RT, and highlight the emerging data for this multimodality approach as well as key preclinical and clinical issues that remain to be addressed. With regards to SCLC, trials exploring to the combination of immunotherapy and RT are already ongoing, but clinical studies for this combination in thymoma are lacking.
\end{abstract}

Keywords: Immunotherapy; radiation; small cell lung cancer (SCLC); thymoma

Submitted Nov 22, 2016. Accepted for publication Mar 15, 2017.

doi: 10.21037/tlcr.2017.03.04

View this article at: http://dx.doi.org/10.21037/tlcr.2017.03.04

\section{Introduction}

Immunotherapy in the context of tumors is a treatment designed to enhance the ability of the immune system to identify and eradicate tumor cells. There has been ample recent evidence to suggest that immunotherapy offers a promising approach for the treatment of several malignancies, notably including solid tumors such as advanced melanoma, renal cell carcinoma, non-small cell lung cancer (NSCLC), as well as hematologic malignancies. Multiple clinical studies are ongoing to examine how best to utilize this promising treatment modality in the context of standards of care (1-4).

The role of the immune system in recognizing and attacking tumor cells has been extensively reviewed in numerous reports elsewhere, most recently by Khalil and colleagues in an excellent overview (5). In brief, it involves the uptake and processing of tumor antigens by antigen-presenting cells (APCs) to activate CD4+ helper T-cells and the ensuing recruitment of CD8+ cytotoxic T-cells to directly kill tumor cells. However, both nontumor immunomodulatory cells within the tumor microenvironment and tumor cells can directly exert an immunosuppressive role to allow the tumor to evade the immune system. One of these mechanisms is the utilization of checkpoint pathways that otherwise are important to protect normal tissue from inflammatory responses. These result in inhibition of anti-tumor T-cell activation. One of the most promising novel treatment strategies, so-called checkpoint blockade, has been demonstrated to block 
this immunosuppression using specific inhibitors against checkpoint receptors such as programmed death 1 (PD-1) on cytotoxic T-cells or programmed death-ligand 1 (PDL1) on antigen-presenting or tumor cells, so that T-cell activation can uninhibitedly proceed and elicit anti-tumor responses (6). Several other approaches also exist, such as adoptive cell therapy, in which native effector T-cells are harvested, engineered to recognize specific tumor associated antigens, and returned to the patient to elicit the anti-tumor response (7). However, for the purposes of this review, we will primarily focus on checkpoint inhibition and the pre-clinical and clinical data exploring this strategy.

Interest in utilizing radiotherapy and immunotherapy together to exploit synergy of these modalities has grown rapidly (8). Tumor cell death following radiation is known to generate and expose neoantigens, thus modulating the tumor immune microenvironment as well as the systemic immune response (9-12), the topic of several recent reviews (13). Hence, there has been an emergence of trials incorporating RT rationally, combining its immunepotentiating effect with checkpoint blockade to improve anti-tumor immune response locally and systemically, as well as trials utilizing "maintenance" checkpoint blockade after chemoradiation treatment to maintain an immune response. Here, we review the preclinical and emerging clinical rationale for utilizing immunotherapy in SCLC as well as thymic tumors, and review the ongoing clinical studies that utilize immunotherapy in combination with $\mathrm{RT}$, in these two disease areas.

\section{Small-cell lung cancer (SCLC)}

SCLC remains one of the most challenging thoracic malignancies. While great advances have been made in NSCLC in identifying molecular targets (i.e., tumors mutant in epidermal growth factor receptor, anaplastic lymphoma kinase) that have extended survival impressively in some patients, such advances in SCLC have remained difficult. SCLC is aggressive, grows rapidly, and early rapid recurrence and metastasis is a hallmark (14). Only about a third of patients present with limited stage disease (LS-SCLC), defined as disease in the hemithorax that can be encompassed by a single conventional RT field; the remainder have extensive stage disease (ES-SCLC) $(15,16)$.

Treatment options have remained unchanged over many years. Etoposide/platinum doublet therapy is the systemic therapy backbone and standard of care for any SCLC, and the pattern of failure is usually characterized by a good upfront response in most patients and early relapse within the first year (17-19). Modifications in the RT approach have been the only contributing factor to improvement in overall survival. These include the timing of RT (concurrent vs. sequential) and the use of twice-daily RT for LS-SCLC, the addition of prophylactic cranial irradiation for both LS- and ES-SCLC, and the addition of consolidative thoracic RT in ES-SCLC (20-23). Despite these improvements survival is dismal, with median overall survival for LS-SCLC of about 25 months, and for ESSCLC of 10 months; analogous 5 -year rates are $10 \%$, and about $2 \%$ (24). Hence, even modest improvements with novel approaches have great potential to be superior to the current standard of care.

\section{Immunotherapy rationale}

Historically, immunotherapy has been difficult to incorporate in to the treatment of SCLC. There have been a number of negative clinical trials in the relatively recent past, including studies showing that post-treatment maintenance therapy with chemotherapy or biologic agents (alpha and gamma interferons, anti-idiotype BEC2 vaccine) did not improve patient outcomes (25). A dendritic cell-based p53 vaccine showed preliminary evidence of immunogenicity (26) but its company is now obsolete making further development of that vaccine unlikely. An additional study examining 2 anti-idiotype vaccines (11D10 and GD2) was terminated due to lack of drug availability (NCT00045617).

With SCLC specifically, two notable characteristics highlight how checkpoint blockade might be incorporated into treatment. First, SCLC has a high mutational burden, as discovered most comprehensively by whole genome sequencing of a large sample of SCLC patients, nearly all of which were treatment naive (27). Bi-allelic inactivation of TP53 and RB1, sometimes by complex genomic rearrangements, was universal. Furthermore, amongst examined solid tumors, SCLC ranks fourth in the number of somatic mutations, a surrogate for the number of neoantigens that a tumor might present to the host immune system $(28,29)$; the top three in that study were melanoma, NSCLC, and bladder cancer. Promising results with checkpoint blockade with two of those three histologies (notably, melanoma and NSCLC) have been observed, and at least in NSCLC, the mutational load seems to govern sensitivity to PD-1 blockade with improved objective response, durable clinical benefit, and 
progression-free survival being associated with higher non-synonymous mutation burden and higher neoantigen burden (30). Hence, given the high mutational load and presumably neoantigen presentation of SCLC, checkpoint blockade may also be an attractive strategy for SCLC.

Second, there are preclinical and clinical data examining T-cell interactions between SCLC and the host immune system. Some in vitro SCLC cell lines appear to secrete IL-15 and induce CD4+ Treg cell mediated immunosuppression (31) and SCLC tumors often contain few infiltrating lymphoid cells (32). Exactly how this is mediated is not clear, but decreased expression of HLA-class I antigen has been reported (33) and the balance between effector and regulatory T-cells distinguishes ESSCLC from LS-SCLC and predicts recurrence (34). This implies that targeting T-cell suppression may be an attractive strategy, and autologous T-cell infusions in patients with advanced SCLC have shown some preliminary evidence of anti-tumor activity in a small non-randomized cohort study (35).

Additionally, SCLC has long been known to induce paraneoplastic disorders in which the host immune system recognizes and targets antigens present on SCLC tumor cells and also in normal tissue. For example, Lambert Eaton Syndrome results from antibodies directed against shared antigens on SCLC cells and normal neurons, including $\mathrm{HuD}$, $\mathrm{HuC}$, and Hel-N1 (36). It was noted nearly two decades ago in retrospective report that SCLC patients with Lambert-Eaton syndrome had improved OS as compared to those without the syndrome (37). Prospective verification of this is ongoing, but the intermediate report is suggestive of benefit (38).

Despite this suggestive data, a phase II trial in this space examining ipilimumab, an anti-cytotoxic T-lymphocyteassociated protein 4 (CTLA4) monoclonal antibody, in the first-line setting combined with paclitaxel/carboplatin in ES-SCLC showed only a 1-month improvement in immune related progression free survival (accounting for tumor response at index lesions in the face of new lesions), but was otherwise reported as a negative trial (39). The ensuing randomized phase III trial with ipilimumab and platinum/etoposide was negative for OS or PFS gain, and showed no change in objective response rate (ORR) (40).

While disappointing, closer examination might yet provide encouragement. As noted by Riess and colleagues (41), early dropout due to disease progression or toxicity was a major problem. Approximately $15 \%$ of randomly assigned patients did not receive the study drug, and only $13 \%$ of those assigned to receive ipilimumab lived long enough without progression or toxicity to receive it as maintenance. Likewise, in KEYNOTE 028, just over half of the patients with SCLC screened for PD-L1 expression who were eligible for pembrolizumab, a PD-1 blocker, actually received it (42). Still, this produced a response rate (RR) of $29 \%$, impressive for previously treated ESSCLC, and comparable to $19-20 \%$ RR observed with nivolumab (a humanized anti-PD-1 agent) or 29-30\% RR with pembrolizumab in previously treated NSCLC patients (43-45). Hence, overcoming the unique clinical challenges of the ES-SCLC patient population may yet reveal a benefit. As they also state, perhaps priming doses of chemotherapy in ES-SCLC are unable to generate the correct or appropriate level of neoantigen expression to drive functional immunogenicity. Lastly, perhaps a strategy to overcome local microenvironment suppression with anti-PD-1 or anti-PD-L1 antibodies is needed in addition to anti-CTLA-4 targeted therapy in which the priming of cytotoxic $\mathrm{T}$ cells is enhanced.

In support of the PD-1/PD-L1 strategy is data from patient samples that noted SCLC cells did not show activation of the pathway, but instead tumor-infiltrating macrophages and tumor-infiltrating lymphocytes (TILs) were activated in $\leq 50 \%$ of the 94 patient samples analyzed, at least suggesting that evaluation of the tumor microenvironment (and not just the tumor itself) should be included in clinical trials (46).

Furthermore, additional evidence comes from the Checkmate 032 study that examined nivolumab with or without ipilimumab in pretreated SCLC patients with progressive disease (47). A RR of $19-23 \%$ was observed for the combination of nivolumab and ipilimumab compared to $10 \%$ with nivolumab alone, and progression-free survival appears to be improved over nivolumab alone, though this was not the intent of the analysis.

The regimens of single agent and combination therapy with checkpoint inhibitors were tolerable; grade 3 or 4 treatment-related adverse events occurred more often in patients receiving the combination of nivolumab and ipilimumab compared to nivolumab monotherapy. Side effects of nivolumab and pembrolizumab in SCLC were similar to those described in the treatment of other cancers with the possible exception of rare autoimmune events such as limbic encephalitis and myasthenia gravis (MG) (47).

\section{Clinical trials of immunotherapy incorporating radiation in SCLC}

While there are several trials of immunotherapy with 
Table 1 Ongoing studies incorporating RT and immunotherapy in SCLC

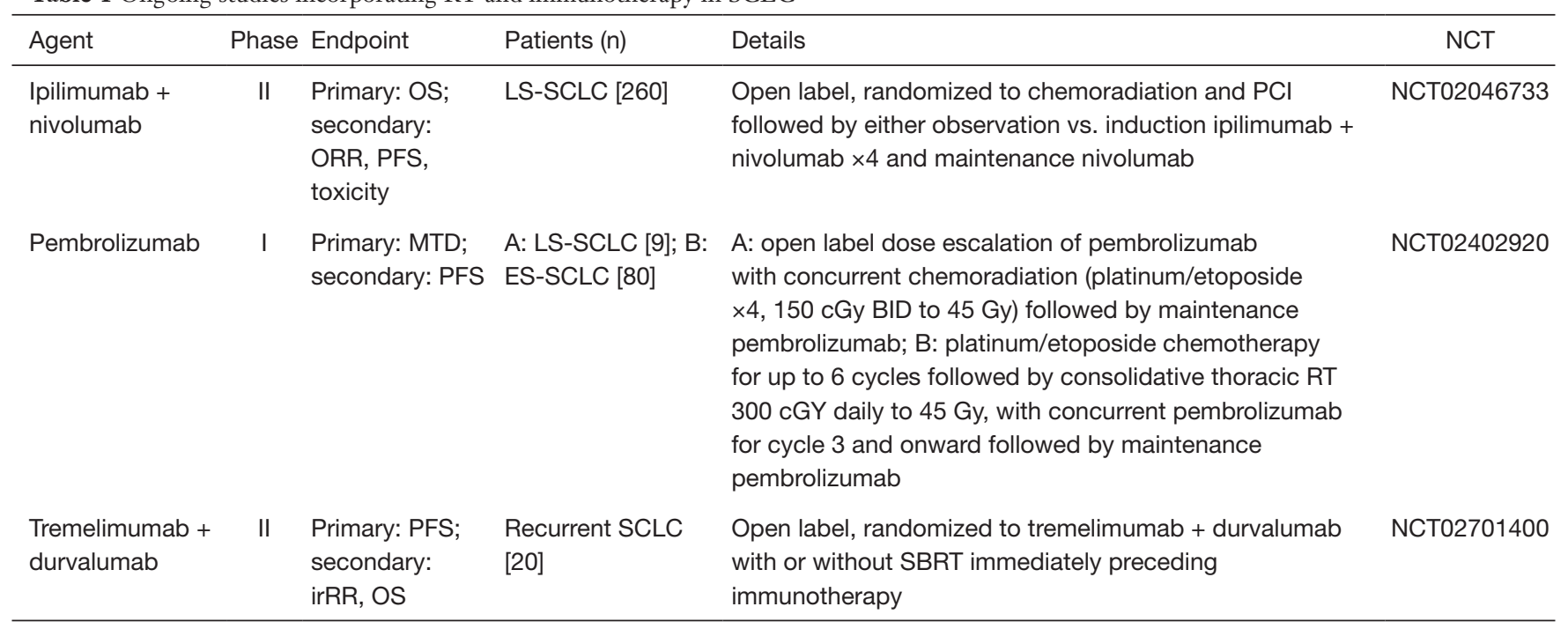

SCLC, small cell lung cancer; OS, overall survival; ORR, objective response rate; PFS, progression-free survival; MTD, maximum tolerated dose; LS-SCLC, limited stage disease; ES-SCLC, extensive stage disease; PCI, prophylactic cranial irradiation; BID, twice daily; SBRT, stereotactic body radiation therapy; NCT, National Clinical Trials number.

systemic therapy in SCLC that have been reviewed just recently (48), here we will focus on the three ongoing studies that include RT (Table 1).

The first focusing on LS-SCLC is the phase II STIMULI trial, incorporating induction with concurrent ipilimumab and nivolumab at about 6-8 weeks following the fourth cycle of standard of care concurrent chemoradiation for LS-SCLC (49). All patients will also undergo prophylactic cranial irradiation following chemoradiation. The induction phase will incorporate four infusions of both checkpoint blockers, to be then followed by maintenance nivolumab alone. The strategy is based on the rationale of combining ipilimumab's peripheral T-cell priming to increase intratumoral $\mathrm{T}$-cells with maintenance PD-1 blockade to then attempt to sustain this activated cell population. One major question will be whether four cycles of chemotherapy with concurrent radiation will lead to suboptimal neoantigen presentation and priming by the time ipilimumab is delivered 2 months following the last dose of therapy, which might not yield the benefit of administrating ipilimumab earlier in the regimen. This study will be open across multiple centers in Europe and is estimated be completed in 2019 .

Similarly, a single-institution phase I dose-escalation study is underway at M.D. Anderson for pembrolizumab given with concurrent RT in two populations (50). The first will include patients with untreated LS-SCLC, and notably, pembrolizumab will be administered upfront with concurrent chemoradiotherapy with platinum/etoposide for standard four cycles, with twice daily RT to the chest in 150 cGy fractions for a total dose of 45 Gy. The second group will include patients with untreated ES-SCLC for whom the treatment paradigm will include pembrolizumab starting with cycle 3 of chemotherapy. RT will be the same dose as the LS-SCLC group, but the timing of this is left open at this point, likely given that the ES-SCLC population will be very heterogeneous and the timing of RT will depend on the response to chemotherapy as well as the functional status of each patient. Given that this is a phase I trial, dose limiting toxicities will be the main focus to judge whether concurrent RT in this potentially ill population will be feasible, with helpful data on pulmonary function and toxicities exacerbated by radiation, but this should provide data for the design of future studies to move checkpoint blockade agents in the first-line setting, and whether twice daily RT in combination with checkpoint inhibition is a manageable strategy.

Both of the above studies will be primarily useful to help judge the effectiveness of the immunotherapy, and perhaps some measures on whether RT augments this in the ES-SCLC population. However, a recently-opened randomized phase II study at Emory University explores whether RT has any impact in eliciting an abscopal effect in SCLC, defined as response in lesions away from that 
which received local therapy. It combines dual checkpoint inhibition using tremelimumab and durvalumab, antiCTLA4 and PD-L1 antibodies respectively, with or without RT (51). In the arm without RT, infusion is every 4 weeks, but in the RT arm, this is preceded by stereotactic body radiation therapy (SBRT) or hypofractionated RT, either daily for 5 days or for three fractions every other day. The primary outcome measure is PFS, and secondary endpoints include the immune-related RR. Crucially, the protocol also specifies paired biopsies at baseline, end of cycle 2, and at progression to characterize evolution in TILs and PD-1/PD-L1 expression, and will allow study for whether this combination approach in SCLC patients shows similar synergistic efficacy as has been seen in melanoma (52).

These are the three main ongoing studies that focus on intersecting RT with immunotherapy in SCLC, and will help to inform basic questions on tolerability, response, and elucidate the intratumoral and circulating host immune responses to immunotherapy and RT combinations. Additional questions on the optimal dose of immunotherapy, dose and fractionation of RT, selection of radiation targets, and how to time the two treatment modalities will remain for future studies. The approach in nearly all cases is empiric, however, there is no solid data at this time to suggest the superiority of one of these combinations over another in any disease, including SCLC.

\section{Thymic tumors}

Thymic tumors represent a heterogeneous group of rare conditions, accounting for less than one percent of all malignancies. Ninety percent of all thymic tumors are thymomas. The 5 -year survival rates for thymomas are well above $70 \%(53,54)$. However, locally-advanced thymomas frequently recur in a more disseminated distribution, mostly in the pleural space, a challenge that is frequently addressed with local therapies such as surgical resection or RT, yet not always with long-term success. For example, in a series of 156 patients with stage II-IV thymoma treated with definitive or adjuvant RT across two large institutions, failures out of the radiation field were the most common, and the five-year failure rate was $24 \%$ (55). Still, postoperative RT in stage II and III thymoma does appear to portend an OS benefit in a large multi-institutional retrospective analysis of 1,263 patients (56).

Thymic carcinomas constitute the most common other histology of thymic tumors, a different disease that is much more aggressive and often treated with tri-modality therapy (57-59). Patients treated with such an aggressive approach appear to have better survival compared with less aggressive treatment among patients with stage III disease (60). In a large international analysis of 1,042 patients with thymic carcinoma, a margin-negative resection and adjuvant RT were important for OS. Still, recurrence rate at 5 years was about $35 \%$ and median OS was 6.6 years (61). Systemic therapy is typically with platinumbased treatment, offering significant disease response and palliation of symptoms in advanced patients (62).

The rare nature of thymic tumors has made largescale preclinical data difficult to generate. This led to the creation of the International Thymic Malignancy Interest Group (ITMIG) and the development of a large, centralized database across 50 institutions with over 6,000 cases at present (63). This collaboration has allowed for consensus guidelines to be developed for classification of thymoma (64), a proposal for standardized TNM staging of thymic tumors $(65,66)$, and response criteria to judge the effectiveness of RT (67).

With regards to immune interactions, thymomas have long been known to interact with T-cell development. For example, an association with MG, a disorder characterized by chronic muscle weakness and antibodies targeting a particular peripheral postsynaptic nicotinic acetylcholine receptor, has been long known. Nearly two decades ago, in a series of 20 patients with thymoma with MG, a higher prevalence of a particular CTLA-4 polymorphic allele with a 3'-untranslated region (AT)n-repeat polymorphism was noted as compared to non-thymoma related MG (68). However, in that study, there was no group of thymoma patients without MG. MG in thymoma seems to be dependent on export of autoreactive CD4+ T-cells, though the exact mechanism still seems to be unclear (69). Associations between CTLA-4 alleles and MG in thymoma patients have been found in populations of Caucasian Germans, as well as Chinese patients $(70,71)$. Still, how this actually impacts the pathogenesis of thymoma, MG, or thymoma-associated MG is not known. Lastly, how CTLA-4 expression impacts patient outcome remains unknown.

Furthermore, the molecular biology of thymic tumors is only just beginning to be understood. Very few cell lines exist; three are from thymic carcinoma patients, and the first reports of $\mathrm{B} 1$ and $\mathrm{AB}$ lines are still relatively recent (72). While genomic aberrations have been known for some time $(73,74)$ in that specifically B3 thymomas display extensive chromosomal imbalances, only one study has performed 
whole genome sequencing (75) and did not identify any mutations of well-characterized cancer genes. Hence, little molecular biology is known about how to integrate immunotherapy approaches for thymomas, for example in the mutational load of thymoma patients.

A few studies have examined the PD-1/PD-L1 pathway in thymic tumors. One Japanese study investigated PD-L1 expression measured by immunohistochemistry and found that PD-L1 expression was significantly higher in thymic carcinomas than in thymomas (76). Data from Stanford University on 69 tumors and 17 normal controls showed strong staining of the epithelial component of thymic tumors, with a higher prevalence in B1-3 thymomas and thymic carcinomas; there was some suggestion of worse prognosis in the PD-L1 positive patients (77). Another study from Japan similarly suggested that PD-L1 expression was associated with more advanced Masaoka stage at presentation and $\mathrm{WHO}$ type $\mathrm{B} 2$ or B3 thymoma, as well as worse disease-free survival (78). The same group also found that 3 of 11 patients with thymic carcinoma showed increase in PD-L1 copy number, and increased expression in these patients correlated with improved OS (79). Lastly, PD-L1 expression was also found in a French series of 104 patients, again confirming the previous data (80).

Another report examining tissue from 15 patients with thymic carcinoma noted strong PD-L1 immunostaining in a third of patient samples (81). Lastly, one anecdotal report also exists showing complete radiographic response to antiPD-1 therapy in a patient with metastatic thymic squamous cell carcinoma (82), as well as one that noted an abscopal response in a patient with thymic carcinoma who was pretreated with granulocyte-macrophage colony-stimulating factor and underwent RT to a single metastasis (83).

Outside of checkpoint blockade studies, alternative immune-directed approaches using specific antigenic targets only recently have begun to show promise. The largest series of thymic epithelial tumors to be examined for expression of mesothelin, a surface antigen present on normal mesothelial cells lining the pleura, peritoneum and pericardium, noted a majority of thymic carcinomas (79\%) and only a small fraction of thymomas (10\%) showed expression (84). In thymic carcinoma, a third showed nearly uniform tumor cell expression, and notably, those with high expression had improved OS. Specific mesothelin-targeted treatment has shown promise in both mesothelioma (with an antimesothelin immunotoxin) as well as advanced pancreatic and ovarian cancer (antibody-drug conjugate), suggesting this may be a promising target in thymic carcinoma as well $(85,86)$.

\section{Clinical trials of immunotherapy in thymic tumors}

Currently there are no clinical trials that combine RT with immunotherapy for thymic tumors. Indeed even those examining immunotherapy alone are few. To our knowledge, only two trials have reported early results with immunotherapy in thymic tumors.

Early results from a phase II study on pembrolizumab in patients with recurrent thymic carcinoma reported that 30 patients showed an objective RR (ORR) of $24 \%$, with five patients with partial response and an additional 10 with stable disease; nearly all of those patients that responded are now beyond 15 cycles of drug (NCT02364076) (87). Of note, an unusual spectrum of autoimmune disorders accompanying this therapy was noted, including polymyositis/myocarditis and type 1 diabetes, manifesting as grade 3 asthenia, complete heart block, and grade 4 hyperglycemia. All recovered with discontinuation of drug and with steroids. Additional studies with pembrolizumab in thymoma are open at MDACC (NCT02721732) and in South Korea (NCT02607631).

Another early report on a phase 1 study of avelumab, a fully human IgG1 anti-PD-L1 antibody, in advanced thymoma (NCT01772004) (88) found that in a cohort of 7 thymoma and 1 thymic carcinoma patients, 4 patients had a partial response (all thymoma), and the only thymic carcinoma patient had stable disease. Similarly as in the study on pembrolizumab, uncommon immune-related adverse events were observed in five patients, including asthenia, myalgia and myositis, among others, and three of the four responding patients had myositis.

Still, immunotherapy in thymoma is only in its infancy. The early preclinical and clinical data appear to be promising, but our understanding of how thymic tumors interface with the host immune system remains poor, largely due to the difficulty of studying the disease owing to its clinical rarity and the availability of few in vitro models. Aside from data on response, these pioneering studies will provide crucial correlative and exploratory data that will allow further investigation of the basic biology of thymic tumors and their interaction with the immune system that will finally allow us to understand this disease better and ultimately provide better specialized and more effective treatment.

\section{Acknowledgements}

Funding: This research was funded in part by the NIH/NCI Cancer Center Support Grant P30 CA008748. 


\section{Footnote}

Conflicts of Interest: The authors have no conflicts of interest to declare.

\section{References}

1. Hodi FS, O'Day SJ, McDermott DF, et al. Improved survival with ipilimumab in patients with metastatic melanoma. N Engl J Med 2010;363:711-23.

2. Maude SL, Frey N, Shaw PA, et al. Chimeric antigen receptor $\mathrm{T}$ cells for sustained remissions in leukemia. $\mathrm{N}$ Engl J Med 2014;371:1507-17.

3. Postow MA, Chesney J, Pavlick AC, et al. Nivolumab and ipilimumab versus ipilimumab in untreated melanoma. $\mathrm{N}$ Engl J Med 2015;372:2006-17.

4. Robert C, Long GV, Brady B, et al. Nivolumab in previously untreated melanoma without BRAF mutation. N Engl J Med 2015;372:320-30.

5. Khalil DN, Smith EL, Brentjens RJ, et al. The future of cancer treatment: immunomodulation, CARs and combination immunotherapy. Nat Rev Clin Oncol 2016;13:273-90.

6. Pardoll DM. The blockade of immune checkpoints in cancer immunotherapy. Nat Rev Cancer 2012;12:252-64.

7. Restifo NP, Dudley ME, Rosenberg SA. Adoptive immunotherapy for cancer: harnessing the $\mathrm{T}$ cell response. Nat Rev Immunol 2012;12:269-81.

8. Reynders K, Illidge T, Siva S, et al. The abscopal effect of local radiotherapy: using immunotherapy to make a rare event clinically relevant. Cancer Treat Rev 2015;41:503-10.

9. Kroemer G, Galluzzi L, Kepp O, et al. Immunogenic cell death in cancer therapy. Annu Rev Immunol 2013;31:51-72.

10. Lee Y, Auh SL, Wang Y, et al. Therapeutic effects of ablative radiation on local tumor require $\mathrm{CD} 8+\mathrm{T}$ cells: changing strategies for cancer treatment. Blood 2009;114:589-95.

11. Lugade AA, Moran JP, Gerber SA, et al. Local radiation therapy of B16 melanoma tumors increases the generation of tumor antigen-specific effector cells that traffic to the tumor. J Immunol 2005;174:7516-23.

12. Reits EA, Hodge JW, Herberts CA, et al. Radiation modulates the peptide repertoire, enhances MHC class I expression, and induces successful antitumor immunotherapy. J Exp Med 2006;203:1259-71.

13. Kalbasi A, June CH, Haas N, et al. Radiation and immunotherapy: a synergistic combination. J Clin Invest 2013;123:2756-63.

14. Rudin CM, Ismaila N, Hann CL, et al. Treatment of Small-Cell Lung Cancer: American Society of Clinical Oncology Endorsement of the American College of Chest Physicians Guideline. J Clin Oncol 2015;33:4106-11.

15. Chan BA, Coward JI. Chemotherapy advances in smallcell lung cancer. J Thorac Dis 2013;5 Suppl 5:S565-78.

16. Samson DJ, Seidenfeld J, Simon GR, et al. Evidence for management of small cell lung cancer: ACCP evidencebased clinical practice guidelines (2nd edition). Chest 2007;132:314S-23S.

17. Hanna N, Bunn PA, Jr., Langer C, et al. Randomized phase III trial comparing irinotecan/cisplatin with etoposide/cisplatin in patients with previously untreated extensive-stage disease small-cell lung cancer. J Clin Oncol 2006;24:2038-43.

18. Hurwitz JL, McCoy F, Scullin P, et al. New advances in the second-line treatment of small cell lung cancer. Oncologist 2009;14:986-94.

19. Sundstrøm S, Bremnes RM, Kaasa S, et al. Cisplatin and etoposide regimen is superior to cyclophosphamide, epirubicin, and vincristine regimen in small-cell lung cancer: results from a randomized phase III trial with 5 years' follow-up. J Clin Oncol 2002;20:4665-72.

20. Aupérin A, Arriagada R, Pignon JP, et al. Prophylactic cranial irradiation for patients with small-cell lung cancer in complete remission. Prophylactic Cranial Irradiation Overview Collaborative Group. N Engl J Med 1999;341:476-84.

21. Aupérin A, Le Péchoux C, Rolland E, et al. Meta-analysis of concomitant versus sequential radiochemotherapy in locally advanced non-small-cell lung cancer. J Clin Oncol 2010;28:2181-90.

22. Turrisi AT, 3rd, Kim K, Blum R, et al. Twice-daily compared with once-daily thoracic radiotherapy in limited small-cell lung cancer treated concurrently with cisplatin and etoposide. N Engl J Med 1999;340:265-71.

23. Slotman BJ, van Tinteren H, Praag JO, et al. Use of thoracic radiotherapy for extensive stage small-cell lung cancer: a phase 3 randomised controlled trial. Lancet 2015;385:36-42.

24. Lally BE, Urbanic JJ, Blackstock AW, et al. Small cell lung cancer: have we made any progress over the last 25 years? Oncologist 2007;12:1096-104.

25. Rossi A, Garassino MC, Cinquini M, et al. Maintenance or consolidation therapy in small-cell lung cancer: a systematic review and meta-analysis. Lung Cancer 
2010;70:119-28.

26. Chiappori AA, Soliman H, Janssen WE, et al. INGN-225: a dendritic cell-based p53 vaccine (Ad.p53-DC) in small cell lung cancer: observed association between immune response and enhanced chemotherapy effect. Expert Opin Biol Ther 2010;10:983-91.

27. George J, Lim JS, Jang SJ, et al. Comprehensive genomic profiles of small cell lung cancer. Nature 2015;524:47-53.

28. Peifer M, Fernández-Cuesta L, Sos ML, et al. Integrative genome analyses identify key somatic driver mutations of small-cell lung cancer. Nat Genet 2012;44:1104-10.

29. Alexandrov LB, Nik-Zainal S, Wedge DC, et al. Signatures of mutational processes in human cancer. Nature 2013;500:415-21.

30. Rizvi NA, Hellmann MD, Snyder A, et al. Cancer immunology. Mutational landscape determines sensitivity to PD-1 blockade in non-small cell lung cancer. Science 2015;348:124-8.

31. Wang W, Hodkinson P, McLaren F, et al. Small cell lung cancer tumour cells induce regulatory $\mathrm{T}$ lymphocytes, and patient survival correlates negatively with FOXP3+ cells in tumour infiltrate. Int J Cancer 2012;131:E928-37.

32. Wang W, Hodkinson P, McLaren F, et al. Histologic assessment of tumor-associated CD45(+) cell numbers is an independent predictor of prognosis in small cell lung cancer. Chest 2013;143:146-51.

33. Tsuchida T, Yamane H, Ochi N, et al. Cytotoxicity of activated natural killer cells and expression of adhesion molecules in small-cell lung cancer. Anticancer Res 2012;32:887-92.

34. Koyama K, Kagamu H, Miura S, et al. Reciprocal CD4+ T-cell balance of effector CD62Llow CD4+ and CD62LhighCD25+CD4+ regulatory T cells in small cell lung cancer reflects disease stage. Clin Cancer Res 2008;14:6770-9.

35. Ding X, Cao H, Chen X, et al. Cellular immunotherapy as maintenance therapy prolongs the survival of the patients with small cell lung cancer. J Transl Med 2015;13:158.

36. Darnell RB. Onconeural antigens and the paraneoplastic neurologic disorders: at the intersection of cancer, immunity, and the brain. Proc Natl Acad Sci U S A 1996;93:4529-36.

37. Maddison P, Newsom-Davis J, Mills KR, et al. Favourable prognosis in Lambert-Eaton myasthenic syndrome and small-cell lung carcinoma. Lancet 1999;353:117-8.

38. Maddison P, Lang B. Paraneoplastic neurological autoimmunity and survival in small-cell lung cancer. J
Neuroimmunol 2008;201-202:159-62.

39. Reck M, Bondarenko I, Luft A, et al. Ipilimumab in combination with paclitaxel and carboplatin as first-line therapy in extensive-disease-small-cell lung cancer: results from a randomized, double-blind, multicenter phase 2 trial. Ann Oncol 2013;24:75-83.

40. Reck M, Luft A, Szczesna A, et al. Phase III Randomized Trial of Ipilimumab Plus Etoposide and Platinum Versus Placebo Plus Etoposide and Platinum in Extensive-Stage Small-Cell Lung Cancer. J Clin Oncol 2016.

41. Riess JW, Lara PN, Jr., Gandara DR. Theory Meets Practice for Immune Checkpoint Blockade in Small-Cell Lung Cancer. J Clin Oncol 2016.

42. Ott PA, Elez E, Hiret S, et al. editors. Pembrolizumab for ED SCLC: Efficacy and Relationship with PD-L1 Expression. IASLC 16th World Conference on Lung Cancer; 2015; Denver, CO.

43. Borghaei H, Paz-Ares L, Horn L, et al. Nivolumab versus Docetaxel in Advanced Nonsquamous Non-Small-Cell Lung Cancer. N Engl J Med 2015;373:1627-39.

44. Brahmer J, Reckamp KL, Baas P, et al. Nivolumab versus Docetaxel in Advanced Squamous-Cell Non-Small-Cell Lung Cancer. N Engl J Med 2015;373:123-35.

45. Herbst RS, Baas P, Kim DW, et al. Pembrolizumab versus docetaxel for previously treated, PD-L1-positive, advanced non-small-cell lung cancer (KEYNOTE-010): a randomised controlled trial. Lancet 2016;387:1540-50.

46. Schultheis AM, Scheel AH, Ozretić L, et al. PD-L1 expression in small cell neuroendocrine carcinomas. Eur J Cancer 2015;51:421-6.

47. Antonia SJ, López-Martin JA, Bendell J, et al. Nivolumab alone and nivolumab plus ipilimumab in recurrent smallcell lung cancer (CheckMate 032): a multicentre, openlabel, phase 1/2 trial. Lancet Oncol 2016;17:883-95.

48. Horn L, Reck M, Spigel DR. The Future of Immunotherapy in the Treatment of Small Cell Lung Cancer. Oncologist 2016;21:910-21.

49. NCT02046733: Small Cell Lung Carcinoma Trial With Nivolumab and IpiliMUmab in Limited Disease (STIMULI). Available online: https://clinicaltrials.gov/ ct2/show/record/NCT02046733

50. NCT02402920: Phase I Trial of MK-3475 and Concurrent Chemo/Radiation for the Elimination of Small Cell Lung Cancer. Available online: https://clinicaltrials.gov/ct2/ show/NCT02402920

51. NCT02701400: Tremelimumab and Durvalumab With or Without Radiation Therapy in Patients With Relapsed Small Cell Lung Cancer. Available online: https:// 
clinicaltrials.gov/ct2/show/NCT02701400

52. Twyman-Saint Victor C, Rech AJ, Maity A, et al. Radiation and dual checkpoint blockade activate non-redundant immune mechanisms in cancer. Nature 2015;520:373-7.

53. Detterbeck FC, Zeeshan A. Thymoma: current diagnosis and treatment. Chin Med J (Engl) 2013;126:2186-91.

54. Detterbeck F, Parsons A. Thymic tumors: a review of current diagnosis, classification, and treatment. In: Patterson G, Cooper J, Deslauriers J, et al. editors. Thoracic and Esophageal Surgery, 3rd Ed. Philadelphia: Elsevier; 2008:1589-614.

55. Rimner A, Gomez DR, Wu AJ, et al. Failure patterns relative to radiation treatment fields for stage II-IV thymoma. J Thorac Oncol 2014;9:403-9.

56. Rimner A, Yao X, Huang J, et al. Postoperative Radiation Therapy Is Associated with Longer Overall Survival in Completely Resected Stage II and III ThymomaAn Analysis of the International Thymic Malignancies Interest Group Retrospective Database. J Thorac Oncol 2016;11:1785-92.

57. Ogawa K, Toita T, Uno T, et al. Treatment and prognosis of thymic carcinoma: a retrospective analysis of 40 cases. Cancer 2002;94:3115-9.

58. Eng TY, Fuller CD, Jagirdar J, et al. Thymic carcinoma: state of the art review. Int J Radiat Oncol Biol Phys 2004;59:654-64.

59. Riely GJ, Huang J, Rimner A. Multidisciplinary management of thymic carcinoma. Am Soc Clin Oncol Educ Book 2012:466-70.

60. Modh A, Rimner A, Allen PK, et al. Treatment Modalities and Outcomes in Patients With Advanced Invasive Thymoma or Thymic Carcinoma: A Retrospective Multicenter Study. Am J Clin Oncol 2016;39:120-5.

61. Ahmad U, Yao X, Detterbeck F, et al. Thymic carcinoma outcomes and prognosis: results of an international analysis. J Thorac Cardiovasc Surg 2015;149:95-100, 1 e1-2.

62. Evans TL, Lynch TJ. Role of chemotherapy in the management of advanced thymic tumors. Semin Thorac Cardiovasc Surg 2005;17:41-50.

63. Huang J, Ahmad U, Antonicelli A, et al. Development of the international thymic malignancy interest group international database: an unprecedented resource for the study of a rare group of tumors. J Thorac Oncol 2014;9:1573-8.

64. Marx A, Ströbel P, Badve SS, et al. ITMIG consensus statement on the use of the WHO histological classification of thymoma and thymic carcinoma: refined definitions, histological criteria, and reporting. J Thorac Oncol 2014;9:596-611.

65. Nicholson AG, Detterbeck FC, Marino M, et al. The IASLC/ITMIG Thymic Epithelial Tumors Staging Project: proposals for the $\mathrm{T}$ Component for the forthcoming (8th) edition of the TNM classification of malignant tumors. J Thorac Oncol 2014;9:S73-80.

66. Kondo K, Van Schil P, Detterbeck FC, et al. The IASLC/ITMIG Thymic Epithelial Tumors Staging Project: proposals for the $\mathrm{N}$ and $\mathrm{M}$ components for the forthcoming (8th) edition of the TNM classification of malignant tumors. J Thorac Oncol 2014;9:S81-7.

67. Benveniste MF, Korst RJ, Rajan A, et al. A practical guide from the International Thymic Malignancy Interest Group (ITMIG) regarding the radiographic assessment of treatment response of thymic epithelial tumors using modified RECIST criteria. J Thorac Oncol 2014;9:S119-24.

68. Huang D, Liu L, Norén K, et al. Genetic association of Ctla-4 to myasthenia gravis with thymoma. J Neuroimmunol 1998;88:192-8.

69. Marx A, Müller-Hermelink HK, Ströbel P. The role of thymomas in the development of myasthenia gravis. Ann N Y Acad Sci 2003;998:223-36.

70. Sun L, Meng Y, Xie Y, et al. CTLA4 variants and haplotype contribute genetic susceptibility to myasthenia gravis in northern Chinese population. PLoS One 2014;9:e101986.

71. Chuang WY, Ströbel P, Gold R, et al. A CTLA4high genotype is associated with myasthenia gravis in thymoma patients. Ann Neurol 2005;58:644-8.

72. Gökmen-Polar Y, Sanders KL, Goswami CP, et al. Establishment and characterization of a novel cell line derived from human thymoma $\mathrm{AB}$ tumor. Lab Invest 2012;92:1564-73.

73. Petrini I, Meltzer PS, Zucali PA, et al. Copy number aberrations of BCL2 and CDKN2A/B identified by array-CGH in thymic epithelial tumors. Cell Death Dis 2012;3:e351.

74. Zettl A, Ströbel P, Wagner K, et al. Recurrent genetic aberrations in thymoma and thymic carcinoma. Am J Pathol 2000;157:257-66.

75. Petrini I, Rajan A, Pham T, et al. Whole genome and transcriptome sequencing of a B3 thymoma. PLoS One 2013;8:e60572.

76. Katsuya Y, Fujita Y, Horinouchi H, et al. Immunohistochemical status of PD-L1 in thymoma and thymic carcinoma. Lung Cancer 2015;88:154-9. 
77. Padda SK, Riess JW, Schwartz EJ, et al. Diffuse high intensity PD-L1 staining in thymic epithelial tumors. J Thorac Oncol 2015;10:500-8.

78. Yokoyama S, Miyoshi H, Nishi T, et al. Clinicopathologic and Prognostic Implications of Programmed Death Ligand 1 Expression in Thymoma. Ann Thorac Surg 2016;101:1361-9.

79. Yokoyama S, Miyoshi H, Nakashima K, et al. Prognostic Value of Programmed Death Ligand 1 and Programmed Death 1 Expression in Thymic Carcinoma. Clin Cancer Res 2016;22:4727-34.

80. Rouquette IL, Taranchon-Clermont E, Guilhodes J, et al. PD-L1 expression in thymic epithelial tumors: A comparative analysis. 7th ITMIG Annual Meeeting 2016.

81. Jian H, Yu Y, Shen L, et al. Oral 1.03: Frequent PD-L1 expression in thymic squamous cell carcinoma of Chinese patients. J Thorac Dis 2015;7:AB056.

82. Yang Y, Ding L, Wang P. Dramatic response to antiPD-1 therapy in a patient of squamous cell carcinoma of thymus with multiple lung metastases. J Thorac Dis 2016;8:E535-7.

Cite this article as: Patel SH, Rimner A, Cohen RB. Combining immunotherapy and radiation therapy for small cell lung cancer and thymic tumors. Transl Lung Cancer Res 2017;6(2):186-195. doi: 10.21037/tlcr.2017.03.04
83. Formenti SC, Demaria S. Systemic effects of local radiotherapy. Lancet Oncol 2009;10:718-26.

84. Thomas A, Chen Y, Berman A, et al. Expression of mesothelin in thymic carcinoma and its potential therapeutic significance. Lung Cancer 2016;101:104-10.

85. Hassan R, Miller AC, Sharon E, et al. Major cancer regressions in mesothelioma after treatment with an antimesothelin immunotoxin and immune suppression. Sci Transl Med 2013;5:208ra147.

86. Weekes CD, Lamberts LE, Borad MJ, et al. Phase I Study of DMOT4039A, an Antibody-Drug Conjugate Targeting Mesothelin, in Patients with Unresectable Pancreatic or Platinum-Resistant Ovarian Cancer. Mol Cancer Ther 2016;15:439-47.

87. Giaccone G, Thompson J, Crawford J, et al. A phase II study of pembrolizumab in patients with recurrent thymic carcinoma. 2016 ASCO Annual Meeting 2016.

88. Rajan A, Heery CR, Kim C, et al. Safety and clinical activity of avelumab (MSB0010718C; anti-PD-L1) in patients with advanced thymoma. 7th ITMIG Annual Meeeting 2016. 